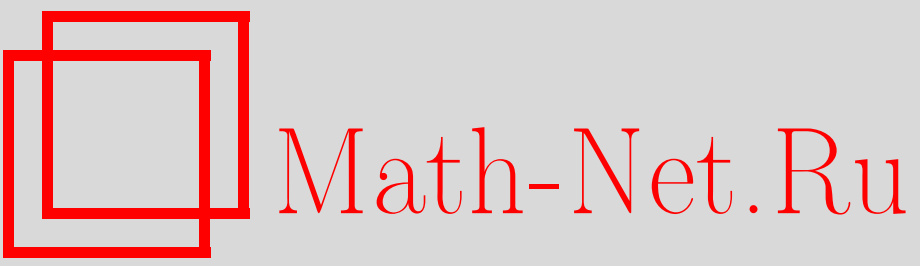

Р. Ю. Леонтьев, Минимальные ветви решений нелинейных операторных уравнений в банаховых пространствах, Итоги науки и техн. Сер. Соврем. мат. и ее прил. Темат. обз., 2020, том 183, 113-119

DOI: https://doi.org/10.36535/0233-6723-2020-183-113-119

Использование Общероссийского математического портала Math-Net.Ru подразумевает, что вы прочитали и согласны с пользовательским соглашением

http://www.mathnet.ru/rus/agreement

Параметры загрузки:

IP : 54.237 .59 .107

26 апреля 2023 г., 15:48:01 
УДК 517.988

\title{
МИНИМАЛЬНЫЕ ВЕТВИ РЕШЕНИЙ НЕЛИНЕЙНЫХ ОПЕРАТОРНЫХ УРАВНЕНИЙ В БАНАХОВЫХ ПРОСТРАНСТВАХ
}

\author{
(C) 2020 г. $\quad$ Р. Ю. ЛЕОНТЬЕВ
}

\begin{abstract}
Аннотация. Рассматривается нелинейное уравнение $B(\lambda) x=R(x, \lambda)+b(\lambda)$, где $R(0,0)=0$, $b(0)=0$, линейный оператор $B(\lambda)$ имеет ограниченный обратный при $S \ni \lambda \rightarrow 0, S$ - некоторое открытое множество, $0 \in \partial S$. Исследуется вопрос существования малого непрерывного решения максимального порядка малости $x(\lambda) \rightarrow 0$ при $S \ni \lambda \rightarrow 0$. Приведен конструктивный способ построения этого решения.
\end{abstract}

Ключевые слова: нелинейный оператор, банахово пространство, операторное уравнение, минимальная ветвь.

\section{MINIMAL BRANCHES OF SOLUTIONS OF NONLINEAR OPERATOR EQUATIONS IN BANACH SPACES}

\author{
(c) 2020 R. YU. LEONTYEV
}

\begin{abstract}
We consider the nonlinear equation $B(\lambda) x=R(x, \lambda)+b(\lambda)$, where $R(0,0)=0, b(0)=0$, the linear operator $B(\lambda)$ has a bounded inverse operator for $S \ni \lambda \rightarrow 0$, and $S$ is an open set, $0 \in \partial S$. We examine the problem on the existence of a small continuous solution of the maximal order od smallness $x(\lambda) \rightarrow 0$ as $S \ni \lambda \rightarrow 0$. A constructive method way of constructing this solution is presented.
\end{abstract}

Keywords and phrases: nonlinear operator, Banach space, operator equation, minimal branch.

AMS Subject Classification: 47J99

Пусть $X, Y$ - банаховы пространства, $\Lambda$ - линейное нормированное пространство. Рассмотрим нелинейное операторное уравнение

$$
B(\lambda) x=R(x, \lambda)+b(\lambda),
$$

где $B(\lambda)$ - замкнутый линейный оператор с плотной в банаховом пространстве $X$ областью определения, не зависящей от параметра $\lambda$. Нелинейный оператор $R(x, \lambda)$ непрерывен по $x$ и $\lambda$ в окрестности точки $(0,0), R(0,0)=0$. Функция $b(\lambda)$ со значениями в $Y$ непрерывна в нуле, $b(0)=0$.

Предположим, что

$$
\left\|B^{-1}(\lambda)\right\|=O\left(\frac{1}{a(\lambda)}\right) \quad x \text { пи } S \ni \lambda \rightarrow 0,
$$

где $a(\lambda)$ - положительный функционал, непрерывный в окрестности нуля, $a(0)=0 ; S \subset \Lambda-$ открытое множество, границе которого принадлежит точка $\lambda=0$. Далее множество $S$ будем называть секториальной окрестностью нуля.

Требуется построить малое решение $x(\lambda) \rightarrow 0$ при $S \ni \lambda \rightarrow 0$ уравнения (1) максимального порядка малости, или «минимальную ветвь».

Впервые теорема о существовании и построении в нерегулярных случаях ветвей решений нелинейных уравнений с векторным параметром в секториальных окрестностях были доказаны в 2004 г. в [5]. Ряд результатов о решениях максимального порядка малости изложен в [1-3]. 
Следует отметить, что оценки вида (2) встречаются в прикладных задачах. Приведем несколько примеров.

Лемма 1. Пусть $X=Y=H, B_{0}$-самосопряженный неотрицательный оператор, $B_{1}-$ самосопряженный положительный оператор, т.е.

$$
\left(B_{0} x, x\right) \geqslant 0, \quad\left(B_{1} x, x\right) \geqslant \gamma(x, x) ; \quad \forall x \in H .
$$

Тогда

$$
\left\|\left(B_{0}+a(\lambda) B_{1}\right)^{-1}\right\|=O\left(\frac{1}{\gamma a(\lambda)}\right) .
$$

Справедливость леммы 1 для компактных операторов следует из теоремы Гильберта-Шмидта о разложении по собственным векторам, а в общем случае - из теории линейных операторов.

Лемма 2. Если элементы $\left\{\varphi_{i}^{(k)}\right\}, k=\overline{1, p_{i}}, i=\overline{1, n}$, образуют полный $B_{1}$-жсорданов набор фредгольмова оператора $B_{0}$ и $p=\max _{i=1, n} p_{i}$, то в некоторой области $0<a(\lambda)<\varepsilon$ существует ограниченный обратный оператор $\left(B_{0}+a(\lambda) B_{1}\right)^{-1}$ и выполнена оценка

$$
\left\|\left(B_{0}+a(\lambda) B_{1}\right)^{-1}\right\|=O\left(\frac{1}{(a(\lambda))^{p}}\right) .
$$

Лемма 3. Пусть нильпотентный оператор $B$ удовлетворяет условию $B^{n}=0$, а оператор $C$ имеет ограниченный обратный оператор, перестановочный с оператором $B, 0<|a(\lambda)|<\varepsilon$. Тогда оператор $а(\lambda) C-B$ имеет ограниченный обратный оператор, для которого выполнена оченка

$$
\left\|(a(\lambda) C-B)^{-1}\right\|=O\left(\left(\frac{1}{|a(\lambda)|}\right)^{n}\right) .
$$

Доказательство. Действительно, убедиться в этом можно, рассмотрев уравнение вида

$$
(a(\lambda) C-B) x=f
$$

или эквивалентное ему уравнение при $\lambda \in S$

$$
x=a^{-1}(\lambda) C^{-1} B x+a^{-1}(\lambda) C^{-1} f .
$$

Далее, формально применяя метод последовательных приближений по формуле

$$
x_{n}=a^{-1}(\lambda) C^{-1} B x_{n-1}+a^{-1}(\lambda) C^{-1} f, \quad x_{0}=0,
$$

на $n$-м шаге получим

$$
x_{n}=a^{-n}(\lambda) C^{-n} B^{n-1} f+a^{-n+1}(\lambda) C^{-n+1} B^{n-2} f+\cdots+a^{-2}(\lambda) C^{-2} B f+a^{-1}(\lambda) C^{-1} f,
$$

которое и будет искомым решением, убедиться в чем можно подстановкой данного выражения в изначальное уравнение.

Далее приведены теоремы, которые дают достаточные условия существования решения уравнения (1), а также описан способ построения этого решения.

Теорема 1. Пусть в секториальной окрестности нуля $S$ для уравнения (1) выполнено условие (2), а также следующие условия:

(1) существует такая непрерывная функиия $\nu(\lambda): S \rightarrow R^{+}, \nu(0)=0$, что в шаре $\|x\| \leqslant r$ при $\lambda \in S$ выполнено неравенство

$$
\left\|R\left(\nu(\lambda) x_{1}, \lambda\right)-R\left(\nu(\lambda) x_{2}, \lambda\right)\right\| \leqslant C \cdot \nu^{l}(\lambda) \cdot r\left\|x_{1}-x_{2}\right\|,
$$

где $l>1, C>0-$ постоянная;

(2) имеет место оценка $\|b(\lambda)\|=o\left(a^{l /(l-1)}(\lambda)\right)$. 
Тогда в некоторой окрестности нуля $\|x\| \leqslant r_{0} \leqslant r$ для любого $\lambda \in S_{0} \subset S$ существует малое решение $x=x^{*}(\lambda)$ уравнения (1), которое является минимальной ветвъю из всех малых решений уравнения (1). Это решение можно найти по формуле

$$
x=a^{1 /(l-1)}(\lambda) y,
$$

где у является пределом последовательности

$$
y_{n}=\left(\frac{1}{a(\lambda)}\right)^{1 /(l-1)} \cdot B^{-1}(\lambda)\left[R\left(a^{1 /(l-1)}(\lambda) y_{n-1}, \lambda\right)+b(\lambda)\right], \quad y_{0}=0 .
$$

Все остальные малье решения уравнения (1) имеют при $S \in \lambda \rightarrow 0$ порлдок малости не выше, чем $a^{1 /(l-1)}(\lambda)$.

Доказательство. Поскольку в области $S$ оператор $B(\lambda)$ имеет ограниченный обратный, то, используя замену $x=\nu(\lambda) y$, приведем уравнение (1) к эквивалентному виду

$$
y=\frac{1}{\nu(\lambda)} B^{-1}(\lambda)[R(\nu(\lambda) y, \lambda)+b(\lambda)] .
$$

Обозначим оператор в правой части последнего выражения через $\Phi(y, \lambda)$. Тогда уравнение примет вид

$$
y=\Phi(y, \lambda)
$$

Покажем, что при выполнении условий теоремы оператор $\Phi(y, \lambda)$ является сжатием.

Учитывая выполнение условия (1) теоремы, имеем

$$
\left\|\Phi\left(y_{1}, \lambda\right)-\Phi\left(y_{2}, \lambda\right)\right\| \leqslant\left\|C \cdot B^{-1}(\lambda) \cdot \nu^{l-1}(\lambda)\right\| \cdot r \cdot\left\|y_{1}-y_{2}\right\| .
$$

Если выбрать функцию $\nu(\lambda)$ так, чтобы $\nu(\lambda)=O\left(a^{1 /(l-1)}(\lambda)\right)$, то будет иметь место неравенство

$$
\left\|C \cdot B^{-1}(\lambda) \cdot \nu^{l-1}(\lambda)\right\| \leqslant C_{1}
$$

где $C_{1}>0$ - постоянная. Зафиксируем $0<q<1$ и выберем радиус $r_{0} \leqslant r$ так, чтобы

$$
r_{0}=\min \left\{\frac{q}{C_{1}}, r\right\} .
$$

Получаем, что оператор $\Phi(y, \lambda)$ является сжатием в шаре $\|y\| \leqslant r_{0}$ при всех $\lambda \in S$ с коэффициентом сжатия $q$.

Далее убедимся, что значения оператора $\Phi(y, \lambda)$ не выходят из шаров радиуса $r_{0}$ для всех элементов $y$, удовлетворяющих условию $\|y\| \leqslant r_{0}$. Учитывая, что оператор $\Phi(y, \lambda)$ является сжатием, получаем

$$
\|\Phi(y, \lambda)\| \leqslant\|\Phi(y, \lambda)-\Phi(0, \lambda)\|+\|\Phi(0, \lambda)\| \leqslant q r_{0}+\left\|\frac{1}{\nu(\lambda)} B^{-1}(\lambda) b(\lambda)\right\|
$$

Так как

$$
\nu(\lambda)=O\left(a^{1 /(l-1)}(\lambda)\right), \quad\left\|B^{-1}(\lambda)\right\|=O b(1 / a(\lambda)),
$$

то второе слагаемое в последнем выражении будет сколь угодно малой величиной при $S \ni \lambda \rightarrow 0$, если $\|b(\lambda)\|=o\left(a^{l /(l-1)}(\lambda)\right)$. Таким образом, если $\|b(\lambda)\|=o\left(a^{l /(l-1)}(\lambda)\right)$, то существует такая секториальная окрестность нуля $S_{0} \subseteq S$, что при всех $\lambda \in S_{0}$ и всех $y$, удовлетворяющих условию $\|y\| \leqslant r_{0}$ будет выполнено неравенство

$$
\|\Phi(y, \lambda)\| \leqslant q r_{0}+(1-q) r_{0}=r_{0}
$$

Теорема доказана.

Необходимо отметить, что в данной теореме не требуется условие существования производной Фреше оператора $R(x, \lambda)$, однако если такая производная существует и является непрерывной по $x$ и $\lambda$ в окрестности нуля, то получаем следующий результат:

Теорема 2. Пусть для уравнения (1) в секториальной окрестности нуля $S$ имеет место оценка (2) и выполнены следующие условия: 
(1) существует непрерывная в нуле производная Фреше по первому аргументу нелинейного оператора $R(x, \lambda)$ и имеет место оченка

$$
\left\|R_{x}(x, \lambda)\right\|=O\left(\|x\|^{l}\right) \quad \text { xnpu }\|x\| \rightarrow 0, l>0 ;
$$

(2) имеет место оценка

$$
\|b(\lambda)\|=o\left(a^{\frac{l}{l-1}}(\lambda)\right) .
$$

Тогда в некоторой окрестности нуля $\|x\| \leqslant r_{0} \leqslant r$ для всех $\lambda \in S_{0} \subset S$ существует малое решение $x=x^{*}(\lambda)$ уравнения (1), которое является минимальной ветвъю из всех малых решений. Это решение можно найти по формуле

$$
x=a^{\frac{1}{l}}(\lambda) y,
$$

где у является пределом последовательности

$$
y_{n}=\left(\frac{1}{a(\lambda)}\right)^{1 / l} \cdot B^{-1}(\lambda)\left[R\left(a^{\frac{1}{l}}(\lambda) y_{n-1}, \lambda\right)+b(\lambda)\right], \quad y_{0}=0 .
$$

Все остальные малье решения уравнения (1) имеют порядок не выше, чем $a^{1 / l}(\lambda)$.

Доказательство. Аналогично тому, как это было сделано в теореме 1, приведем уравнение (1) к виду (3). Для этого воспользуемся обратимостью оператора $B^{-1}(\lambda)$ в области $S$ и заменой $x=\nu(\lambda) y$, где параметр $\nu(\lambda)$ - непрерывный функционал $\lambda$, условия на который будут получены исходя из условий принципа сжимающих отображений. Применяя оценку нормы произведения, интегральную теорему Лагранжа и используя первое условие теоремы 2 , получим следующую цепочку неравенств:

$$
\begin{gathered}
\left\|\Phi\left(y_{1}, \lambda\right)-\Phi\left(y_{2}, \lambda\right)\right\|=\left\|\frac{1}{\nu(\lambda)} B^{-1}(\lambda)\left[R\left(\nu(\lambda) y_{1}, \lambda\right)-R\left(\nu(\lambda) y_{2}, \lambda\right)\right]\right\| \leqslant \\
\leqslant\left\|\frac{1}{\nu(\lambda)} B^{-1}(\lambda)\right\| \cdot\left\|\int_{0}^{1} R_{x}\left(\nu(\lambda)\left(y_{2}+\Theta\left(y_{1}-y_{2}\right)\right), \lambda\right) d \Theta \cdot \nu(\lambda)\left(y_{1}-y_{2}\right)\right\| \leqslant \\
\leqslant\left\|B^{-1}(\lambda)\right\| \cdot \int_{0}^{1} C|\nu(\lambda)|^{l}\left\|y_{2}+\Theta\left(y_{1}-y_{2}\right)\right\|^{l} d \Theta \cdot\left\|y_{1}-y_{2}\right\| \leqslant \\
\leqslant C\left\|B^{-1}(\lambda)\right\| \cdot|\nu(\lambda)|^{l} \int_{0}^{1}\left[\left\|y_{1}\right\|+\Theta\left(\left\|y_{1}\right\|+\left\|y_{2}\right\|\right)\right]^{l} d \Theta\left\|y_{1}-y_{2}\right\| .
\end{gathered}
$$

Здесь $C$ - некоторая константа, которая неизбежно возникает в процессе оценки, но она не влияет на дальнейшие рассуждения. Выберем в качестве $\nu(\lambda)$ любое выражение, удовлетворяющее условию $\nu(\lambda)=O\left(a^{1 / l}(\lambda)\right)$ при $\lambda \rightarrow 0$. Кроме того, используем условие, что все оценки производятся в шаре $\|y\| \leqslant r$. Получим

$$
\left\|\Phi\left(y_{1}, \lambda\right)-\Phi\left(y_{2}, \lambda\right)\right\| \leqslant C_{1} \cdot r^{l} \int_{0}^{1}[1+2 \Theta]^{l} d \Theta\left\|y_{1}-y_{2}\right\| .
$$

Константа $C_{1}$ появилась после сокращения выражений $\left\|B^{-1}(\lambda)\right\|$ и $|\nu(\lambda)|^{l}$, которые после введения условия на $\nu(\lambda)$ в виде оценки, зависящей от $a(\lambda)$, стали иметь порядки $O(1 / a(\lambda))$ и $O(a(\lambda))$ соответственно. После вычисления интеграла получаем

$$
\left\|\Phi\left(y_{1}, \lambda\right)-\Phi\left(y_{2}, \lambda\right)\right\| \leqslant C_{1} \cdot r^{l} \frac{1}{2(l+1)}\left\|y_{1}-y_{2}\right\| .
$$

Зафиксируем $0<q<1$ и выберем $r_{0}=\min \left\{r, 2 q(l+1) /\left(C_{1} r^{l-1}\right)\right\}$. Тогда оператор $\Phi(y, \lambda)$ является сжимающим в шаре $\|y\| \leqslant r_{0}$ при всех $\lambda \in S$. 
Теперь необходимо проверить, что значения оператора $\Phi(y, \lambda)$ не выходят из шаров $\|y\| \leqslant r_{0}$. Но поскольку условие сжимаемости оператора $\Phi(y, \lambda)$ выполнено, а условие $(2)$ теоремы 2 такое же, как и в теореме 1 , то доказательство того, что $\|\Phi(y, \lambda)\| \leqslant r_{0}$ в некоторой секториальной окрестности нуля $S_{0} \subseteq S$ будет таким же, как для теоремы 1 . Поэтому опустим эту часть доказательства.

После того, как мы показали, что к уравнению (3) можно применять принцип сжимающих отображений, будем искать решение в виде предела последовательности $\left\{y_{n}\right\}, n=1,2, \ldots$, где $y_{n}=\Phi\left(y_{(n-1)}, \lambda\right)$, а в качестве начального приближения выберем $y_{0}=0$ или любой другой элемент из шара $\|y\| \leqslant r_{0}$. Тот факт, что предел последовательности существует, гарантируется принципом сжимающих отображений. Поэтому решение уравнения (3) обозначим через $y=\lim _{n \rightarrow \infty} y_{n}$. Но тогда решение уравнения (1) будет иметь вид $x=a^{1 / l}(\lambda) y$. Кроме указанного решения уравнения (1) в общем случае могут существовать и другие малые решения уравнения (1), поскольку уравнение (1) в силу оценки (2) не относится к регулярному случаю. Но из всех малых решений найденное нами решение будет решением максимального порядка малости.

Замечание 1. Приведенные выше примеры показывают, что теоремы 1,2 позволяют строить решения минимального порядка малости нелинейных уравнений второго и первого рода.

Пример 1. Рассмотрим задачу

$$
\left\{\begin{array}{l}
\lambda \frac{\partial^{2} u(t, x)}{\partial t^{2}}+\int_{a}^{b} K(x, s) u(t, s) d s=f(u(t, x), t, x, \lambda), \\
\left.u(t, x)\right|_{t=0}=\left.u(t, x)\right|_{t=1}=0 .
\end{array}\right.
$$

Здесь $\lambda \in \mathbb{R} ; t, x \in \mathbb{R} ; u(t, x)$ - непрерывная, дважды непрерывно дифференцируемая по $t$ функция, обращающаяся в нуль при $t=0$ и $t=1 ; f$ - нелинейная непрерывная функция.

Введем обозначения:

$$
B u=\int_{a}^{b} K(x, s) u(t, s) d s, \quad C u=\left\{\begin{array}{l}
\frac{\partial^{2} u(t, x)}{\partial t^{2}}, \\
\left.u(t, x)\right|_{t=0}=\left.u(t, x)\right|_{t=1}=0 .
\end{array}\right.
$$

Пусть ядро $K(x, s)$ оператора $B$ удовлетворяет условию

$$
\int_{a}^{b} K(x, z) K(z, t) d z=0
$$

тогда оператор $B$ является нильпотентным и удовлетворяет условию $B^{2}=0$. Примером оператора, удовлетворяющего условию (4), может служить, например, функция вида $K(x, s)=\cos x \cdot \sin s$ при $a=0, b=\pi$.

Краевая задача

имеет единственное непрерывное решение вида

$$
\left\{\begin{array}{l}
\frac{\partial^{2} u(t, x)}{\partial t^{2}}=m(t, x) \\
\left.u(t, x)\right|_{t=0}=\left.u(t, x)\right|_{t=1}=0
\end{array}\right.
$$

$$
u(t, x)=\int_{0}^{1} G(t, s) m(s, x) d s
$$

где функция Грина имеет вид

$$
G(t, x)= \begin{cases}t(s-1), & 0 \leqslant t \leqslant s \\ (t-1) s, & s \leqslant t \leqslant 1\end{cases}
$$


Следовательно, оператор $C$ имеет ограниченный обратный оператор вида

$$
C^{-1} u=\int_{0}^{1} G(t, s) u(s, x) d s,
$$

перестановочный с оператором $B$.

Согласно введенным выше обозначениям, исходное уравнение имеет вид

$$
(\lambda C+B) u=f .
$$

Из леммы 3 следует, что оператор $\lambda C+B$, стоящий в левой части данного уравнения, имеет ограниченный обратный, для которого выполнена оценка

$$
\left\|(\lambda C+B)^{-1}\right\|=O\left(|\lambda|^{-2}\right) .
$$

Пусть функция $f$ удовлетворяет всем условиям теоремы 2 , а именно, пусть функция $f(u(t, x), t, x, \lambda)$ имеет непрерывную производную по первому аргументу, для которой выполняется условие

$$
\left\|f_{u}(u(t, x), t, x, \lambda)\right\|=O\left(\|u(t, x)\|^{l}\right) \quad \text { при }\|u(t, x)\| \rightarrow 0, l>0,
$$

а также имеет место оценка

$$
\|f(0, t, x, \lambda)\|=o\left(\lambda^{(l+1) / l}\right) .
$$

Тогда будут выполнены все условия теоремы 2 , и в некотором шаре $\|u\| \leqslant r_{0}$ для любого $\lambda$ из проколотой окрестности нуля $S_{0}$ существует малое решение данного уравнения, которое является минимальной ветвью из всех малых решений. Укажем формулу для поиска этого решения методом последовательных приближений. Для этого найдем в явном виде выражение для оператора $(\lambda C+B)^{-1}$, для чего воспользуемся формулой, полученной в лемме 3. Поскольку в нашем случае $n=2$, то данная формула будет иметь вид

$$
u=\lambda^{-1} C^{-1} f-\lambda^{-2} C^{-2} B f .
$$

Согласно конкретному виду операторов $C^{-1}$ и $B$, имеем

$$
\begin{aligned}
C^{-2} u=\int_{0}^{1} G(t, s) \int_{0}^{1} G(s, z) u(z, x) d z d s= \\
=\int_{0}^{1}\left(\int_{0}^{1} G(t, s) G(s, z) d s\right) u(z, x) d z=\int_{0}^{1} G_{1}(t, z) u(z, x) d z, \\
C^{-2} B f u=\int_{0}^{1} G_{1}(t, z) \int_{a}^{b} K(x, s) f(u(z, s), z, s, \lambda) d s d z, \\
C^{-1} f u=\int_{0}^{1} G(t, s) f(u(s, x), s, x, \lambda) d s d z .
\end{aligned}
$$

Тогда, согласно формуле (6), исходное уравнение примет вид

$$
u=\frac{1}{\lambda} \int_{0}^{1} G(t, s) f(u(s, x), s, x, \lambda) d s-\frac{1}{\lambda^{2}} \int_{0}^{1} G_{1}(t, z) \int_{a}^{b} K(x, s) f(u(z, s), z, s, \lambda) d s d z
$$

и по теореме 2 минимальную ветвь можно найти по формуле

$$
u(t, x)=\lambda^{\frac{1}{l}} v(t, x),
$$


где $v(t, x)$ является пределом последовательности:

$$
\begin{aligned}
v_{n}(t, x)=\left(\frac{1}{\lambda}\right)^{1+1 / l} & \int_{0}^{1} G(t, s) f\left(\lambda^{1 / l} v_{n-1}(s, x), s, x, \lambda\right) d s- \\
& -\left(\frac{1}{\lambda}\right)^{2+1 / l} \int_{0}^{1} G_{1}(t, z) \int_{a}^{b} K(x, s) f\left(\lambda^{1 / l} v_{n-1}(z, s), z, s, \lambda\right) d s d z, \quad v_{0}=0
\end{aligned}
$$

\section{СПИСОК ЛИТЕРАТУРЫ}

1. Леонтъев Р. Ю. О решениях максимального порядка малости нелинейных уравнений// в кн.: Наука в вузах: Математика, физика, информатика. Проблемы высшего и среднего профессионального образования. - М.: РУДН, 2009. - С. 276-278.

2. Леонтъев Р. Ю. О решениях максимального порядка малости нелинейных уравнений// Вестн. Бурят. гос. ун-та. Мат. информ. - 2009. - № 9. - С. 77-83.

3. Леонтъев P. Ю., Сидоров Н. А. Униформизация и последовательные приближения решений нелинейных уравнений с векторным параметром// Изв. Иркутск. гос. ун-та. Мат. — 2011. - 4, № 3. C. $116-123$

4. Леонтъев P. Ю. О малых решениях нелинейных операторных уравнений с векторным параметром// Мат. XII Междунар. науч.-техн. конф. «Математическое и компьютерное моделирование естественнонаучных и социальных проблем». - Пенза: Изд-во ПГУ, 2018. - С. 80-83.

5. Сидоров H. А. Минимальные ветви решений нелинейных уравнений и асимптотические регуляризаторы// Нелин. гранич. задачи. - 2004. - № 14. - С. 161-164.

Леонтьев Роман Юрьевич

Иркутский государственный университет

E-mail: romanisu@yandex.ru 